\title{
Controllability of Damped Second-Order Neutral Impulsive Stochastic Functional Differential Systems with Infinite Delay
}

\author{
R.Sathya and K.Balachandran* \\ Department of Mathematics, Bharathiar University, Coimbatore - 641046. \\ Email: sathyain.math@gmail.com
}

\begin{abstract}
In this paper we study the controllability of damped second order neutral impulsive stochastic functional differential system with infinite delay in Hilbert spaces. Sufficient conditions for controllability results are obtained by using the theory of cosine families of bounded linear operators and fixed point technique. An example is provided to illustrate the theory.
\end{abstract}

Keywords: Controllability, Second order damped stochastic system, Neutral impulsive functional differential system, Cosine family, Fixed point.

\section{Introduction}

Second order systems capture the dynamic behavior of many natural phenomena and have found applications in several fields such as mathematical physics, biology and finance. A variety of problems arising in mechanics, elasticity theory, molecular dynamics, and quantum mechanics can be described in general by nonlinear partial differential equations of second order in time. Damping literally means the action of a substance or an element in a mechanical or electrical device that gradually reduces the degree of oscillation, vibration, or signal intensity or prevent it from increasing. For example, sound-proofing technology dampens the oscillations of sound waves. Damping may be mathematically modeled as a force synchronous with the velocity of the object but opposite in direction to it. Examples are the viscous regularization of the Sine- or Klein-Gordon equation [1], the equations describing a vibrating membrane or a vibrating nonlocal beam [1], the equations describing phase transformations in shape-memory alloys [2], and further the equations in thermo-viscoelasticity. The functional analytic formulation of the foregoing problems leads to initial value problems of the form

$$
u^{\prime \prime}+A u^{\prime}+B u=f \quad \text { in }(0, T), \quad u(0)=u_{0}, \quad u^{\prime}(0)=v_{0},
$$

where $A$ and $B$ are nonlinear operators defined on appropriate spaces.

Consider a nonlinear wave equation with damping as in [3]

$$
\begin{aligned}
& \frac{\partial^{2} u}{\partial t^{2}}-M\left(\int_{\Omega}|\nabla u(x, t)|^{2} d x\right) \triangle u+(-\triangle)^{\alpha} \frac{\partial u}{\partial t}=f \text { in } Q, \text { with } 0<\alpha \leq 1, \\
& u(x, t)=0 \text { for }(x, t) \in \Sigma, \\
& u(x, 0)=u_{0}(x), \frac{\partial u}{\partial t}(x, 0)=u_{1}(x) \text { in } \Omega,
\end{aligned}
$$

where $M(s)$ is a positive continuous function on $[0, \infty), \Omega$ is a bounded open set of $\mathbb{R}^{n}$ with smooth boundary $\Gamma, Q$ is the cylinder $\Omega \times(0, \infty)$ of $\mathbb{R}^{n+1}$, with lateral boundary $\Sigma=\Gamma \times(0, \infty) ; \triangle=\sum_{i=1}^{n} \frac{\partial^{2}}{\partial x_{i}^{2}}$ is the Laplace operator and $|\nabla u(x, t)|^{2}=\sum_{i=1}^{n}\left|\frac{\partial u}{\partial x_{i}}\right|^{2}$. Equation (1.2) without damping $(-\triangle)^{\alpha} \frac{\partial u}{\partial t}$ occurs in the study of vibrations of an elastic string. The above equation can be written in the abstract form as

$$
\begin{aligned}
& u^{\prime \prime}+M\left(\left|A^{1 / 2} u\right|^{2}\right) A u+A^{\alpha} u^{\prime}=f \text { in }(0, T), 0<\alpha \leq 1, \\
& u(0)=u_{0}, \quad u^{\prime}(0)=u_{1} .
\end{aligned}
$$

Equations (1.3) is similar to (1.1) if $M\left(\left|A^{1 / 2} u\right|^{2}\right) A=B$ and $\alpha=1$. The deterministic version of second order differential equations have been thoroughly investigated by several authors $[4,5]$ while the stochastic 
version has been in progressing state. In many cases, it is advantageous to treat the second-order stochastic differential equations directly rather than to convert them to first-order equations. The second-order stochastic differential equations are the right model in continuous time to account for integrated processes that can be made stationary. For instance, it is useful for engineers to model mechanical vibrations or charge on a capacitor or condenser subjected to white noise excitation through a second-order stochastic differential equations.

Neutral differential equations have many applications in physical and biological systems, for this reason those equations have received much attention among researchers. The neutral stochastic differential equations can play an important role in describing many sophisticated dynamical systems in physical, biological, medical, chemical engineering, aero-elasticity etc. Stochastic differential equations (SDEs) with finite delay arise naturally in the mathematical modeling of various phenomena in natural and social sciences. When the delay is infinite, the situation is more complicated, since the properties of solutions depend on the choice of the phase space $\mathcal{B}$. The book [6] contains the fundamental theory related to functional differential equations with infinite delay. Several works have been established in this direction, we refer to $[7,8,9,10,11,12]$.

The study of impulsive functional differential equations is linked to their utility in stimulating processes and phenomena subject to short-time perturbations during their evolution. The perturbations are performed discretely and their duration is negligible in comparison with the total duration of the process. The theory of impulsive differential and functional differential equations have been extensively developed. Delay differential equations involving impulsive effects occur in many applications such as population dynamics, biology, ecology, epidemics, etc (see $[13,14]$ ).

The literatures regarding second order neutral and impulsive functional differential equations are very extensive (see $[15,16,17]$ ). Mahmudov and Mckibben $[18,19]$ derived the existence and controllabilty of second-order neutral stochastic evolution equation by using cosine family theory. Sakthivel [20] studied controllability of second order nonlinear impulsive differential systems. Hernandez et al. [21] have explored the existence results for a damped second order abstract functional differential equation with impulses. Mahmudov and McKibben [22] discussed the abstract second order damped Mckean-Vlasov stochastic evolution equations. Mckibben [23] have investigated second order damped functional stochastic evolution equations in Hilbert spaces. More recently, Arthi and Balachandran [24] derived the controllability of damped second order impulsive neutral functional differential systems with infinite delay. As far as we know, there is no work reported on the controllability of damped second order stochastic functional differential systems with infinite delay in the phase space $\mathcal{B}$ and the objective of this paper is to fill this gap. Moreover, the present work is the stochastic extension of the work [24].

\section{Preliminaries}

Consider the following class of damped second order neutral impulsive stochastic functional differential system with infinite delay

$$
\begin{aligned}
\mathrm{d}\left[x^{\prime}(t)-g\left(t, x_{t}\right)\right]= & {\left[A x(t)+\mathcal{D} x^{\prime}(t)+B u(t)+f\left(t, x_{t}\right)\right] \mathrm{d} t } \\
& \quad+\sigma\left(t, x_{t}\right) \mathrm{d} w(t), t \in J:=[0, a], t \neq t_{k}, \\
x_{0}=\phi \in \mathcal{B}, \quad x^{\prime}(0)=\eta \in H, & =I_{k}\left(x_{t_{k}}\right), \quad k=1,2, \cdots, m, \\
\triangle x\left(t_{k}\right)= & J_{k}\left(x_{t_{k}}\right), \quad k=1,2, \cdots, m,
\end{aligned}
$$

where the state variable $x(\cdot)$ takes values in a real separable Hilbert space $H$ with innerproduct $(\cdot, \cdot)$ and norm $\|\cdot\|$ and $A$ is the infinitesimal generator of a strongly continuous cosine family of bounded linear operators $\{C(t): t \in \mathbb{R}\}$ defined on $H$. The control function $u(\cdot)$ takes values in $L^{2}(J, U)$ of admissible control functions for a separable Hilbert space $U, B$ is a bounded linear operator from $U$ into $H$ and $\mathcal{D}$ is a bounded linear operator on $H$ with $D(\mathcal{D}) \subset D(A)$. Let $K$ be another separable Hilbert space with innerproduct $(\cdot, \cdot)_{K}$ and the norm $\|\cdot\|_{K}$. Suppose $\{w(t): t \geq 0\}$ is a given $K$-valued Wiener process with a finite trace nuclear covariance operator $Q \geq 0$. We employ the same notation $\|\cdot\|$ for the norm $\mathcal{L}(K, H)$, where $\mathcal{L}(K, H)$ denotes the space of all bounded linear operators from $K$ into $H$. Further, 
$g: J \times \mathcal{B} \rightarrow H, f: J \times \mathcal{B} \rightarrow H$ and $\sigma: J \times \mathcal{B} \rightarrow \mathcal{L}_{Q}(K, H)$ are measurable mappings in $H$-norm and $\mathcal{L}_{Q}(K, H)$-norm respectively. Here $\mathcal{L}_{Q}(K, H)$ denotes the space of all $Q$-Hilbert-Schmidt operators from $K$ into $H$ which will be defined in Section 2. The functions $I_{k}, J_{k}: \mathcal{B} \rightarrow H(k=1,2, \cdots, m)$ are bounded functions. Furthermore the fixed times $t_{k}$ satisfies $0=t_{0}<t_{1}<t_{2}<\cdots<t_{m}<t_{m+1}=a$ and $\triangle \xi\left(t_{k}\right)=\xi\left(t_{k}^{+}\right)-\xi\left(t_{k}^{-}\right)$represents the jump of a function $\xi$ at $t_{k}$ where $\xi\left(t_{k}^{+}\right)$and $\xi\left(t_{k}^{-}\right)$denote the right and left limits of $\xi$ at $t_{k}$ and $I_{k}, J_{k}$ determines the size of the jump. The histories $x_{t}:(-\infty, 0] \rightarrow H$, $t \geq 0$ defined by setting $x_{t}(\theta)=\{x(t+\theta),-\infty<\theta \leq 0\}$ belongs to some abstract phase space $\mathcal{B}$ defined axiomatically in Section 2. The initial values $\phi, \eta$ are $\mathcal{F}_{0}$-adapted, $\phi$ is a $\mathcal{B}$-valued random variable and $\eta$ is a $H$-valued $\mathcal{F}_{t}$-measurable function independent of $\{w(t): t \geq 0\}$ with finite second moment.

Let $(\Omega, \mathcal{F}, P ; \mathbf{F})\left\{\mathbf{F}=\left\{\mathcal{F}_{t}\right\}_{t \geq 0}\right\}$ be a complete filtered probability space satisfying that $\mathcal{F}_{0}$ contains all $P$-null sets of $\mathcal{F}$. An $H$-valued random variable is an $\mathcal{F}$-measurable function $x(t): \Omega \rightarrow H$ and a collection of random variables $S=\{x(t, \omega): \Omega \rightarrow H \backslash t \in J\}$ is called a stochastic process. Usually, we supress the dependence on $\omega \in \Omega$ and write $x(t)$ instead of $x(t, \omega)$ and $x(t): J \rightarrow H$ in the place of $S$. Suppose $x(t): \Omega \rightarrow H, t \geq-\infty$ is a continuous $\mathcal{F}_{t}$-adapted, $H$-valued stochastic process. We can associate with another process $x_{t}: \Omega \rightarrow \mathcal{B}, t \geq 0$ by setting $x_{t}=\{x(t+s)(\omega): s \in(-\infty, 0]\}$. This is regarded as a $\mathcal{B}$-valued stochastic process. Let $\left\{e_{n}\right\}_{n=1}^{\infty}$ be a complete orthonormal basis of $K$. Suppose that $\{w(t): t \geq 0\}$ is a cylindrical $K$-valued wiener process with a finite trace nuclear covariance operator $Q \geq 0$, denote $\operatorname{Tr}(Q)=\sum_{n=1}^{\infty} \lambda_{n}=\lambda<\infty$, which satisfies that $Q e_{n}=\lambda_{n} e_{n}$. So, actually, $w(t)=\sum_{n=1}^{\infty} \sqrt{\lambda_{n}} \omega_{n}(t) e_{n}$, where $\left\{\omega_{n}(t)\right\}_{n=1}^{\infty}$ are mutually independent one-dimensional standard Wiener processes. We assume that $\mathcal{F}_{t}=\sigma\{w(s): 0 \leq s \leq t\}$ is the $\sigma$-algebra generated by $w$ and $\mathcal{F}_{a}=\mathcal{F}$. Let $\Psi \in \mathcal{L}(K, H)$ and define

$$
\|\Psi\|_{Q}^{2}=\operatorname{Tr}\left(\Psi Q \Psi^{*}\right)=\sum_{n=1}^{\infty}\left\|\sqrt{\lambda_{n}} \Psi e_{n}\right\|^{2} .
$$

If $\|\Psi\|_{Q}<\infty$, then $\Psi$ is called a $Q$-Hilbert-Schmidt operator. Let $\mathcal{L}_{Q}(K, H)$ denote the space of all $Q$-Hilbert-Schmidt operators $\Psi: K \rightarrow H$. The completion $\mathcal{L}_{Q}(K, H)$ of $\mathcal{L}(K, H)$ with respect to the topology induced by the norm $\|\cdot\|_{Q}$ where $\|\Psi\|_{Q}^{2}=\langle\Psi, \Psi\rangle$ is a Hilbert space with the above norm topology. For more details in this section refer Prato [25].

Now we review some basic definitions, notations, properties and fundamental facts needed to establish our result. Throughout this paper, $A$ is the infinitesimal generator of a strongly continuous cosine family $(C(t))_{t \in \mathbb{R}}$ of bounded linear operators on a Hilbert space $(H,\|\cdot\|)$.

Definition: 2.1. A one-parameter family $(C(t))_{t \in \mathbb{R}}$ of bounded linear operators mapping the Hilbert space $H$ into itself is called a strongly continuous cosine family iff

(i) $C(s+t)+C(s-t)=2 C(s) C(t)$, for all $t, s \in \mathbb{R}$,

(ii) $C(0)=I$,

(iii) $C(t) x$ is continuous in $t$ on $\mathbb{R}$ for each fixed $x \in H$.

The strongly continuous sine family $\{S(t): t \in \mathbb{R}\}$, associated to the given strongly continuous cosine family $\{C(t): t \in \mathbb{R}\}$, defined by

$$
S(t) x=\int_{0}^{t} C(s) x \mathrm{~d} s, x \in H, t \in \mathbb{R} .
$$

Moreover, we denote by $N$ and $\tilde{N}$ a pair of positive constants such that $\|C(t)\|^{2} \leq N$ and $\|S(t)\|^{2} \leq \tilde{N}$ for every $t \in J$. The infinitesimal generator of a strongly continuous cosine family $C(t), t \in \mathbb{R}$, is the operator $A: H \rightarrow H$ defined by

$$
A x=\left.\frac{\mathrm{d}^{2}}{\mathrm{~d} t^{2}} C(t) x\right|_{t=0} \quad x \in D(A)
$$

where $D(A)=\{x \in H ; C(t) x$ is twice continuously differentiable in $t\}$.

Define $E=\{x \in H: C(t) x$ is once continuously differentiable in $t\}$.

In this paper, $D(A)$ represents the domain of $A$ endowed with the graph norm $\|x\|_{A}=\|x\|+\|A x\|$, $x \in D(A)$. The notation $E$ stands for the space formed by the vectors $x \in H$ for which $C(\cdot) x$ is of class $C^{1}$ 
on $\mathbb{R}$. It was proved by Kisynski [26] that $E$ endowed with the norm $\|x\|_{E}=\|x\|+\sup _{0 \leq t \leq 1}\|A S(t) x\|, \quad x \in E$, is a Banach space. The operator valued function

$$
\mathcal{H}(t)=\left[\begin{array}{ll}
C(t) & S(t) \\
A S(t) & C(t)
\end{array}\right]
$$

is a strongly continuous group of bounded linear operators on the space $E \times H$ generated by the operator $\mathcal{A}=\left[\begin{array}{ll}0 & I \\ A & 0\end{array}\right]$ defined on $D(A) \times E$. From this, it follows that $A S(t): E \rightarrow H$ is a bounded linear operator and that $\|A S(t) x\| \rightarrow 0$ as $t \rightarrow 0$, for each $x \in E$. Furthermore if $x:[0, \infty) \rightarrow H$ is locally integrable, then $y(t)=\int_{0}^{t} S(t-s) x(s) \mathrm{d} s$ is an $E$-valued continuous function which is a consequence of the fact that

$$
\int_{0}^{t} \mathcal{H}(t-s)\left[\begin{array}{l}
0 \\
x(s)
\end{array}\right] \mathrm{d} s=\left[\begin{array}{l}
\int_{0}^{t} S(t-s) x(s) \mathrm{d} s \\
\int_{0}^{t} C(t-s) x(s) \mathrm{d} s
\end{array}\right]
$$

defines an $E \times H$-valued continuous function.

The existence of solutions for the second order abstract Cauchy problem

$$
\begin{aligned}
x^{\prime \prime}(t) & =A x(t)+h(t), \quad t \in J, \\
x(0) & =z_{0}, \quad x^{\prime}(0)=z_{1},
\end{aligned}
$$

where $h: J \rightarrow H$ is an integrable function, has been discussed in [4]. On the other hand, the semilinear case has been treated in [5]. We only mention here that the function $x: J \rightarrow H$ given by

$$
x(t)=C(t) z_{0}+S(t) z_{1}+\int_{0}^{t} S(t-s) h(s) \mathrm{d} s, \quad t \in J
$$

is called a mild solution of (2.2)-(2.3) and that, when $z_{0} \in E, x(\cdot)$ is continuously differentiable and

$$
x^{\prime}(t)=A S(t) z_{0}+C(t) z_{1}+\int_{0}^{t} C(t-s) h(s) \mathrm{d} s, \quad t \in J .
$$

For additional material related to cosine function theory, we refer to $[4,5,27]$.

The collection of all strongly measurable, square integrable $H$-valued random variables denoted by $\mathcal{L}_{2}(\Omega, \mathcal{F}, P ; H) \equiv \mathcal{L}_{2}(\Omega, H)$, is a Banach space equipped with norm $\|x(\cdot)\|_{\mathcal{L}_{2}}=\left(E\|x(\cdot ; \omega)\|_{H}^{2}\right)^{\frac{1}{2}}$, where the expectation $E$ is defined by $E(h)=\int_{\Omega} h(\omega) d P$. Similarly, $\mathcal{L}_{2}^{\mathcal{F}}(\Omega, H)$ denotes the Banach space of all $\mathcal{F}_{t}$-measurable, square integrable random variables, such that $\int_{\Omega}\|x(t, \cdot)\|_{\mathcal{L}_{2}}^{2} \mathrm{~d} t<\infty$. A function $u:[\sigma, \tau] \rightarrow \mathcal{L}_{2}$ is said to be a normalised piecewise continuous function on $[\sigma, \tau]$ if $u$ is piecewise continuous and left continuous on $(\sigma, \tau]$. We denote by $\mathcal{P} C\left([\sigma, \tau], \mathcal{L}_{2}\right)$ the space of normalised piecewise continuous function from $[\sigma, \tau]$ into $\mathcal{L}_{2}$.

In this work, we will employ an axiomatic definition of the phase space $\mathcal{B}$, similar to the one used in [6] and suitably modified to treat retarded impulsive differential equations. Specifically, $\mathcal{B}$ will be a linear space of functions mapping $(-\infty, 0]$ into $H$ endowed with a seminorm $\|\cdot\|_{\mathcal{B}}$ and we will assume that $\mathcal{B}$ satisfies the following axioms:

(A) If $x:(-\infty, \sigma+b] \rightarrow H, b>0, \sigma \in \mathbb{R}$, is such that $x_{\sigma} \in \mathcal{B}$ and $\left.x\right|_{[\sigma, \sigma+b]} \in \mathcal{P} C\left([\sigma, \sigma+b], \mathcal{L}_{2}\right)$, then for every $t \in[\sigma, \sigma+b)$, the following conditions hold:

(i) $x_{t}$ is in $\mathcal{B}$,

(ii) $\|x(t)\| \leq L\left\|x_{t}\right\|_{\mathcal{B}}$,

(iii) $\left\|x_{t}\right\|_{\mathcal{B}} \leq K(t-\sigma) \sup \{\|x(s)\|: \sigma \leq s \leq t\}+M(t-\sigma)\left\|x_{\sigma}\right\|_{\mathcal{B}}$,

where $L>0$ is a constant; $K, M:[0, \infty) \rightarrow[1, \infty), K$ is continuous, $M$ is locally bounded and $L, K, M$ are independent of $x(\cdot)$.

(B) The space $\mathcal{B}$ is complete.

Remark: 2.1. In impulsive functional differential systems, the map $[\sigma, \sigma+b] \rightarrow \mathcal{B}, t \rightarrow x_{t}$, is in general discontinuous. For this reason, this property has been omitted from our description of the phase space $\mathcal{B}$. 
Next we consider an example of a phase space.

Example: 2.1. The phase space $\mathcal{P C}_{r} \times \mathcal{L}^{p}(\rho, H)$

Let $r \geq 0,1 \leq p<\infty$ and let $\rho:(-\infty,-r] \rightarrow \mathbb{R}$ be a non-negative measurable function which satisfies the conditions $(\mathrm{g}-5),(\mathrm{g}-6)$ in the terminology of [6]. Briefly, this means that $\rho$ is locally integrable and there exists a non-negative, locally bounded function $\gamma$ on $(-\infty, 0]$ such that $\rho(\xi+\theta) \leq \gamma(\xi) \rho(\theta)$, for all $\xi \leq 0$ and $\theta \in(-\infty,-r) \backslash N_{\xi}$, where $N_{\xi} \subseteq(-\infty,-r)$ is a set with Lebesgue measure zero. The space $\mathcal{B}=\mathcal{P C}_{r} \times \mathcal{L}^{p}(\rho, H)$ consists of all classes of Lebesgue-measurable functions $\varphi:(-\infty, 0] \rightarrow H$ such that $\left.\varphi\right|_{[-r, 0]} \in \mathcal{P C}([-r, 0], H)$ and $\rho\|\varphi\|^{p}$ is Lebesgue integrable on $(-\infty,-r)$. The seminorm in this space is defined by

$$
\|\varphi\|_{\mathcal{B}}=\sup \{\|\varphi(\theta)\|:-r \leq \theta \leq 0\}+\left(\int_{-\infty}^{-r} \rho(\theta)\|\varphi(\theta)\|^{p} d \theta\right)^{1 / p} .
$$

Proceeding as in the proof of [6, Theorem 1.3.8], it follows that $\mathcal{B}$ is a space which satisfies the axioms (A) and (B). Moreover, when $r=0$ this space coincides with $C_{0} \times \mathcal{L}^{p}(\rho, H)$ and if, in addition, $p=2$, we can take $L=1, M(t)=\gamma(-t)^{1 / 2}$ and $K(t)=1+\left(\int_{-t}^{0} \rho(\theta) d \theta\right)^{1 / 2}$ for $t \geq 0$.

Denote $J_{0}=\left[0, t_{1}\right], J_{k}=\left(t_{k}, t_{k+1}\right], k=1,2, \cdots, m$, and define the following class of functions: $\mathcal{P} C\left(J, \mathcal{L}_{2}(\Omega, H)\right)=\left\{x: J \rightarrow \mathcal{L}_{2}:\left.x\right|_{\left(t_{k}, t_{k+1}\right]} \in \mathcal{C}\left(J_{k}, \mathcal{L}_{2}\right), k=0,1,2, \cdots, m\right.$ and there exist $x\left(t_{k}^{-}\right)$and $x\left(t_{k}^{+}\right)$with $\left.x\left(t_{k}^{-}\right)=x\left(t_{k}\right), k=1,2,3, \cdots, m\right\}$ is the Banach space of normalised piecewise continuous maps from $J$ into $\mathcal{L}_{2}(\Omega, H)$ satisfying the condition $\sup _{t \in J} E\|x(t)\|^{2}<\infty$. Let $\mathcal{Z} \equiv \mathcal{P} C\left(J, \mathcal{L}_{2}\right)$ be the closed subspace of $\mathcal{P} C\left(J, \mathcal{L}_{2}^{\mathcal{F}}(\Omega, H)\right)$ consisting of measurable, $\mathcal{F}_{t}$ - adapted and $H$-valued processes $x(t)$. Then $\mathcal{Z}$ is a Banach space endowed with the norm

$$
\|x\|_{\mathcal{Z}}=\left(\sup _{t \in J} E\left\|x_{t}\right\|_{\mathcal{B}}^{2}\right)^{\frac{1}{2}}
$$

where

$$
\left\|x_{t}\right\|_{\mathcal{B}} \leq M_{a} E\|\phi\|_{\mathcal{B}}+K_{a} \sup \{E\|x(s)\|: 0 \leq s \leq a\},
$$

$M_{a}=\sup \{M(t-\sigma): t \in[\sigma, \sigma+a]\}, K_{a}=\sup \{K(t-\sigma): t \in[\sigma, \sigma+a]\}$. Let $J_{1}=(-\infty, a]$. Now we define the mild solution of (2.1) as in [28].

Definition: 2.2. A stochastic process $x$ is said to be a mild solution of (2.1) if the following conditions are satisfied:

(a) $x(t, \omega)$ is a measurable function from $J_{1} \times \Omega$ to $H$ and $x(t)$ is $\mathcal{F}_{t}$-adapted for all $t \in J_{1}$,

(b) $E\|x(t)\|^{2}<\infty$ for each $t \in J_{1}$,

(c) $\triangle x\left(t_{k}\right)=I_{k}\left(x_{t_{k}}\right), \triangle x^{\prime}\left(t_{k}\right)=J_{k}\left(x_{t_{k}}\right), \quad k=1,2, \cdots, m$,

(d) For each $u \in L_{2}^{\mathcal{F}}(J, U)$, the process $x$ satisfies the following integral equation

$$
\begin{aligned}
x(t)= & C(t) \phi(0)+S(t)[\eta-g(0, \phi)]+\int_{0}^{t} C(t-s) g\left(s, x_{s}\right) \mathrm{d} s \\
& +\sum_{i=0}^{j-1}\left[S\left(t-t_{i+1}\right) \mathcal{D} x\left(t_{i+1}^{-}\right)-S\left(t-t_{i}\right) \mathcal{D} x\left(t_{i}^{+}\right)\right]-S\left(t-t_{j}\right) \mathcal{D} x\left(t_{j}^{+}\right) \\
& +\int_{0}^{t} C(t-s) \mathcal{D} x(s) \mathrm{d} s+\int_{0}^{t} S(t-s)\left[B u(s)+f\left(s, x_{s}\right)\right] \mathrm{d} s \\
& +\int_{0}^{t} S(t-s) \sigma\left(s, x_{s}\right) \mathrm{d} w(s)+\sum_{0<t_{k}<t} C\left(t-t_{k}\right) I_{k}\left(x_{t_{k}}\right) \\
& +\sum_{0<t_{k}<t} S\left(t-t_{k}\right) J_{k}\left(x_{t_{k}}\right), \quad \text { for a.e. } t \in\left[t_{j}, t_{j+1}\right], j=0,1,2, \cdots, m, \\
x_{0}= & \phi \in \mathcal{L}_{2}(\Omega, \mathcal{B}), \quad x^{\prime}(0)=\eta \in \mathcal{L}_{2}(\Omega, H) .
\end{aligned}
$$


Definition: 2.3. [15] The system (2.1) is said to be controllable on the interval $J$, if for every $\phi \in \mathcal{B}$ with $\phi(0) \in D(A), \eta \in E$ and $x_{1} \in H$, there exists a control $u \in L^{2}(J, U)$ such that the solution $x(\cdot)$ of (2.1) satisfies $x(a)=x_{1}$.

In order to establish our controllability result we assume the following hypotheses:

(H1) The linear operator $W: L^{2}(J, U) \rightarrow H$ defined by

$$
W u=\int_{0}^{a} S(a-s) B u(s) \mathrm{d} s
$$

is invertible with inverse operator $W^{-1}$ taking values in $L^{2}(J, U) \backslash k e r W$ and there exist positive constants $N_{B}, N_{W}$ such that

$$
\|B\|^{2} \leq N_{B},\left\|W^{-1}\right\|^{2} \leq N_{W} .
$$

(H2) There exists a constant $N_{D}>0$ such that $\|\mathcal{D}\|^{2} \leq N_{D}$.

(H3) (i) The function $g: J \times \mathcal{B} \rightarrow H$ is continuous and there exists a constant $N_{g}>0$ for $t, s \in J$ and $\gamma, \xi \in \mathcal{B}$ such that

$$
E\|g(t, \gamma)-g(s, \xi)\|^{2} \leq N_{g}\left(|t-s|^{2}+\|\gamma-\xi\|_{\mathcal{B}}^{2}\right)
$$

(ii) There exist constants $N_{1}>0$ and $N_{2}>0$ such that

$$
E\|g(t, \gamma)\|^{2}=N_{1}\|\gamma\|_{\mathcal{B}}^{2}+N_{2}
$$

where $N_{2}=\sup _{t \in J}\|g(t, 0)\|^{2}$.

(H4) The nonlinear function $f: J \times \mathcal{B} \rightarrow H$ is Lipschitz continuous and there exist constants $N_{f}>$ $0, \tilde{N}_{f}>0$ for $t \in J, \gamma, \xi \in \mathcal{B}$ such that

$$
E\|f(t, \gamma)-f(t, \xi)\|^{2} \leq N_{f}\|\gamma-\xi\|_{\mathcal{B}}^{2}
$$

and $\tilde{N}_{f}=\sup _{t \in J}\|f(t, 0)\|^{2}$.

(H5) The function $\sigma: J \times \mathcal{B} \rightarrow \mathcal{L}_{Q}(K, H)$ is continuous and there exist constants $N_{\sigma}>0, \tilde{N}_{\sigma}>0$ for $t \in J$ and $\gamma, \xi \in \mathcal{B}$ such that

$$
E\|\sigma(t, \gamma)-\sigma(t, \xi)\|_{Q}^{2} \leq N_{\sigma}\|\gamma-\xi\|_{\mathcal{B}}^{2}
$$

and $\tilde{N}_{\sigma}=\sup _{t \in J}\|\sigma(t, 0)\|^{2}$.

(H6) $I_{k}, J_{k}: \mathcal{B} \rightarrow H$ are continuous and there exist positive constants $\beta_{k}, \alpha_{k}, \tilde{\beta}_{k}, \tilde{\alpha}_{k}>0$ such that

$$
\begin{aligned}
& E\left\|I_{k}(\gamma)-I_{k}(\xi)\right\|^{2} \leq \beta_{k}\|\gamma-\xi\|_{\mathcal{B}}^{2}, \quad k=1,2, \cdots, m, \\
& E\left\|J_{k}(\gamma)-J_{k}(\xi)\right\|^{2} \leq \alpha_{k}\|\gamma-\xi\|_{\mathcal{B}}^{2}, \quad k=1,2, \cdots, m
\end{aligned}
$$

and $\tilde{\beta}_{k}=\left\|I_{k}(0)\right\|^{2}, \tilde{\alpha_{k}}=\left\|J_{k}(0)\right\|^{2}, k=1,2, \cdots, m$.

(H7) There exists a constant $\nu>0$ such that

$$
\begin{aligned}
\nu=9 K_{a}^{2}\left\{( 1 + 8 a ^ { 2 } \tilde { N } N _ { B } N _ { W } ) \left(a^{2} N N_{g}+\frac{1}{K_{a}^{2}}\left(5 \tilde{N}+a^{2} N\right) N_{D}+a^{2} \tilde{N} N_{f}\right.\right. \\
\left.\left.+a \tilde{N} T r(Q) N_{\sigma}+m N \sum_{k=1}^{m} \beta_{k}+m \tilde{N} \sum_{k=1}^{m} \alpha_{k}\right)\right\}<1 \\
\text { and } L=10\left\{( 1 + 1 2 a ^ { 2 } \tilde { N } N _ { B } N _ { W } ) \left[\tilde{N}\left(N_{1}\|\widehat{\phi}\|_{\mathcal{B}}^{2}+N_{2}\right)+a^{2} N\left(4 N_{1} N^{*}+N_{2}\right)\right.\right. \\
+\left(10 \tilde{N}+2 a^{2} N\right) N_{D}\|\widehat{\phi}\|_{a}+2 a^{2} \tilde{N}\left[4 N_{f} N^{*}+\tilde{N}_{f}\right]+2 a \tilde{N} \operatorname{Tr}(Q)\left[4 N_{\sigma} N^{*}\right. \\
\left.\left.+\tilde{N}_{\sigma}\right]+2 m N\left[4 \sum_{k=1}^{m} \beta_{k} N^{*}+\sum_{k=1}^{m} \tilde{\beta}_{k}\right]+2 m \tilde{N}\left[4 \sum_{k=1}^{m} \alpha_{k} N^{*}+\sum_{k=1}^{m} \tilde{\alpha}_{k}\right]\right] \\
\left.+12 a^{2} \tilde{N} N_{B} N_{W}\left[\left\|x_{1}\right\|^{2}+N\|\phi(0)\|^{2}+\tilde{N}\|\eta\|^{2}\right]\right\} \\
\text { where } N^{*}=2 K_{a}^{2}\left(N\|\phi(0)\|^{2}+\tilde{N}\|\eta\|^{2}\right)+M_{a}^{2}\|\widehat{\phi}\|_{\mathcal{B}}^{2} .
\end{aligned}
$$




\section{Controllability Result}

Theorem: 3.1. If the conditions $(H 1)-(H 7)$ hold then the system $(2.1)$ is controllable on $J$ provided that

$$
\begin{aligned}
10\{(1+ & \left.12 a^{2} \tilde{N} N_{B} N_{W}\right) 4 K_{a}^{2}\left(a^{2} N N_{1}+\frac{N_{D}}{2 K_{a}^{2}}\left(5 \tilde{N}+a^{2} N\right)+2 a^{2} \tilde{N} N_{f}+2 a \tilde{N} \operatorname{Tr}(Q) N_{\sigma}\right. \\
& \left.\left.+2 m\left[N \sum_{k=1}^{m} \beta_{k}+\tilde{N} \sum_{k=1}^{m} \alpha_{k}\right]\right)\right\}<1 .
\end{aligned}
$$

Proof: For an arbitrary function $x(\cdot)$ we define the control using the hypothesis $(H 1)$

$$
\begin{aligned}
u_{x}^{a}(t)= & W^{-1}\left[x_{1}-C(a) \phi(0)-S(a)[\eta-g(0, \phi)]-\int_{0}^{a} C(a-s) g\left(s, x_{s}\right) \mathrm{d} s\right. \\
& -\sum_{i=0}^{j-1}\left[S\left(a-t_{i+1}\right) \mathcal{D} x\left(t_{i+1}^{-}\right)-S\left(a-t_{i}\right) \mathcal{D} x\left(t_{i}^{+}\right)\right]+S\left(a-t_{j}\right) \mathcal{D} x\left(t_{j}^{+}\right) \\
& -\int_{0}^{a} C(a-s) \mathcal{D} x(s) \mathrm{d} s-\int_{0}^{a} S(a-s) f\left(s, x_{s}\right) \mathrm{d} s-\int_{0}^{a} S(a-s) \sigma\left(s, x_{s}\right) \mathrm{d} w(s) \\
& \left.-\sum_{0<t_{k}<a} C\left(a-t_{k}\right) I_{k}\left(x_{t_{k}}\right)-\sum_{0<t_{k}<a} S\left(a-t_{k}\right) J_{k}\left(x_{t_{k}}\right)\right](t) .
\end{aligned}
$$

Consider the space $\mathcal{B}_{a}=\left\{x: J_{1} \rightarrow H: x_{0}=\phi \in \mathcal{B}\right.$ and $\left.\left.x\right|_{J} \in \mathcal{P} C\left(J, \mathcal{L}_{2}\right)\right\}$. Let $\|\cdot\|_{a}$ be a seminorm in $B_{a}$ defined by

$$
\|x\|_{a}=\left\|x_{0}\right\|_{\mathcal{B}}+\sup _{0 \leq s \leq a}\left(E\|x(s)\|^{2}\right)^{1 / 2}, x \in \mathcal{B}_{a}
$$

Define the operator $\Psi: \mathcal{B}_{a} \rightarrow \mathcal{B}_{a}$ by

$$
(\Psi x)(t)=\left\{\begin{array}{l}
\phi(t), \quad t \in(-\infty, 0] \\
C(t) \phi(0)+S(t)[\eta-g(0, \phi)]+\int_{0}^{t} C(t-s) g\left(s, x_{s}\right) \mathrm{d} s \\
+\sum_{i=0}^{j-1}\left[S\left(t-t_{i+1}\right) \mathcal{D} x\left(t_{i+1}^{-}\right)-S\left(t-t_{i}\right) \mathcal{D} x\left(t_{i}^{+}\right)\right]-S\left(t-t_{j}\right) \mathcal{D} x\left(t_{j}^{+}\right) \\
+\int_{0}^{t} C(t-s) \mathcal{D} x(s) \mathrm{d} s+\int_{0}^{t} S(t-s)\left[B u(s)+f\left(s, x_{s}\right)\right] \mathrm{d} s \\
+\int_{0}^{t} S(t-s) \sigma\left(s, x_{s}\right) \mathrm{d} w(s)+\sum_{0<t_{k}<t} C\left(t-t_{k}\right) I_{k}\left(x_{t_{k}}\right) \\
+\sum_{0<t_{k}<t} S\left(t-t_{k}\right) J_{k}\left(x_{t_{k}}\right), \quad \text { for a.e. } t \in J .
\end{array}\right.
$$

We shall show that the operator $\Psi$ has a fixed point, which is then a solution of system (2.1). Clearly, $(\Psi x)(a)=x_{1}$. For $\phi \in \mathcal{B}$, define

$$
\widehat{\phi}(t)= \begin{cases}\phi(t), & t \in(-\infty, 0], \\ C(t) \phi(0)+S(t) \eta, & t \in J,\end{cases}
$$


then $\widehat{\phi}(t) \in \mathcal{B}_{a}$. Set $x(t)=z(t)+\widehat{\phi}(t), \quad t \in J_{1}$. It is clear that $x$ satisfies (2.6) if and only if $z$ satisfies $z_{0}=0$ and

$$
\begin{aligned}
z(t)= & -S(t) g\left(0, \widehat{\phi}_{0}\right)+\int_{0}^{t} C(t-s) g\left(s, z_{s}+\widehat{\phi}_{s}\right) \mathrm{d} s+\int_{0}^{t} S(t-s) B u_{z+\widehat{\phi}}^{a}(s) \mathrm{d} s \\
& +\sum_{i=0}^{j-1}\left[S\left(t-t_{i+1}\right) \mathcal{D}\left(z\left(t_{i+1}^{-}\right)+\widehat{\phi}\left(t_{i+1}^{-}\right)\right)-S\left(t-t_{i}\right) \mathcal{D}\left(z\left(t_{i}^{+}\right)+\widehat{\phi}\left(t_{i}^{+}\right)\right)\right] \\
& -S\left(t-t_{j}\right) \mathcal{D}\left(z\left(t_{j}^{+}\right)+\widehat{\phi}\left(t_{j}^{+}\right)\right)+\int_{0}^{t} C(t-s) \mathcal{D}(z(s)+\widehat{\phi}(s)) \mathrm{d} s \\
& +\int_{0}^{t} S(t-s) f\left(s, z_{s}+\widehat{\phi}_{s}\right) \mathrm{d} s+\int_{0}^{t} S(t-s) \sigma\left(s, z_{s}+\widehat{\phi}_{s}\right) \mathrm{d} w(s) \\
& +\sum_{0<t_{k}<t} C\left(t-t_{k}\right) I_{k}\left(z_{t_{k}}+\widehat{\phi}_{t_{k}}\right)+\sum_{0<t_{k}<t} S\left(t-t_{k}\right) J_{k}\left(z_{t_{k}}+\widehat{\phi}_{t_{k}}\right), \text { where } \\
u_{z+\widehat{\phi}}^{a}(t)= & W^{-1}\left[x_{1}-C(a) \phi(0)-S(a)\left[\eta-g\left(0, \widehat{\phi}_{0}\right)\right]-\int_{0}^{a} C(a-s) g\left(s, z_{s}+\widehat{\phi}_{s}\right) \mathrm{d} s\right. \\
& -\sum_{i=0}^{j-1}\left[S\left(a-t_{i+1}\right) \mathcal{D}\left(z\left(t_{i+1}^{-}\right)+\widehat{\phi}\left(t_{i+1}^{-}\right)\right)-S\left(a-t_{i}\right) \mathcal{D}\left(z\left(t_{i}^{+}\right)+\widehat{\phi}\left(t_{i}^{+}\right)\right)\right] \\
& +S\left(a-t_{j}\right) \mathcal{D}\left(z\left(t_{j}^{+}\right)+\widehat{\phi}\left(t_{j}^{+}\right)\right)-\int_{0}^{a} C(a-s) \mathcal{D}(z(s)+\widehat{\phi}(s)) \mathrm{d} s \\
& -\int_{0}^{a} S(a-s) f\left(s, z_{s}+\widehat{\phi}_{s}\right) \mathrm{d} s-\int_{0}^{a} S(a-s) \sigma\left(s, z_{s}+\widehat{\phi}_{s}\right) \mathrm{d} w(s) \\
& -\sum_{0<t_{k}<a} C\left(a-t_{k}\right) I_{k}\left(z_{t_{k}}+\widehat{\phi}_{t_{k}}\right)-\sum_{0<t_{k}<a} S\left(a-t_{k}\right) J_{k}\left(z_{t_{k}}+\widehat{\phi}_{t_{k}}\right) .
\end{aligned}
$$

Let $\mathcal{B}_{a}^{0}=\left\{y \in \mathcal{B}_{a}: y_{0}=0 \in \mathcal{B}\right\}$. For any $y \in \mathcal{B}_{a}^{0}$, we have

$$
\|y\|_{a}=\left\|y_{0}\right\|_{\mathcal{B}}+\sup _{0 \leq s \leq a}\left(E\|y(s)\|^{2}\right)^{1 / 2}=\sup _{0 \leq s \leq a}\left(E\|y(s)\|^{2}\right)^{1 / 2}
$$

and thus $\left(\mathcal{B}_{a}^{0},\|\cdot\|_{a}\right)$ is a Banach space. Set

$$
\mathcal{B}_{q}=\left\{y \in \mathcal{B}_{a}^{0}: y(0)=0,\|y\|_{a}^{2} \leq q\right\} \text { for some } q \geq 0,
$$

then $\mathcal{B}_{q} \subseteq \mathcal{B}_{a}^{0}$ is a bounded closed convex set, and for $z \in \mathcal{B}_{q}$, we have

$$
\begin{aligned}
\left\|z_{t}+\widehat{\phi}_{t}\right\|_{\mathcal{B}}^{2} & \leq 2\left(\left\|z_{t}\right\|_{\mathcal{B}}^{2}+\left\|\widehat{\phi}_{t}\right\|_{\mathcal{B}}^{2}\right) \\
& \leq 4\left(K_{a}^{2} \sup _{0 \leq s \leq a} E\|z(s)\|^{2}+M_{a}^{2}\left\|z_{0}\right\|_{\mathcal{B}}^{2}+K_{a}^{2} \sup _{0 \leq s \leq a} E\|\widehat{\phi}(t)\|^{2}+M_{a}^{2}\left\|\widehat{\phi}_{0}\right\|_{\mathcal{B}}^{2}\right) \\
& \leq 4\left(K_{a}^{2} q+2 K_{a}^{2}\left(N\|\phi(0)\|^{2}+\tilde{N}\|\eta\|^{2}\right)+M_{a}^{2}\|\widehat{\phi}\|_{\mathcal{B}}^{2}\right) \\
& \leq 4\left(K_{a}^{2} q+N^{*}\right) .
\end{aligned}
$$


Let the operator $\widehat{\Psi}: \mathcal{B}_{a}^{0} \rightarrow \mathcal{B}_{a}^{0}$ defined by

$$
(\widehat{\Psi} x)(t)=\left\{\begin{array}{l}
0, \\
-S(t) g\left(0, \widehat{\phi}_{0}\right)+\int_{0}^{t} C(t-s) g\left(s, z_{s}+\widehat{\phi}_{s}\right) \mathrm{d} s \\
+\sum_{i=0}^{j-1}\left[S\left(t-t_{i+1}\right) \mathcal{D}\left(z\left(t_{i+1}^{-}\right)+\widehat{\phi}\left(t_{i+1}^{-}\right)\right)-S\left(t-t_{i}\right) \mathcal{D}\left(z\left(t_{i}^{+}\right)+\widehat{\phi}\left(t_{i}^{+}\right)\right)\right] \\
-S\left(t-t_{j}\right) \mathcal{D}\left(z\left(t_{j}^{+}\right)+\widehat{\phi}\left(t_{j}^{+}\right)\right)+\int_{0}^{t} C(t-s) \mathcal{D}(z(s)+\widehat{\phi}(s)) \mathrm{d} s \\
+\int_{0}^{t} S(t-s)\left[B u_{z+\widehat{\phi}^{a}}^{a}(s)+f\left(s, z_{s}+\widehat{\phi}_{s}\right)\right] \mathrm{d} s \\
+\int_{0}^{t} S(t-s) \sigma\left(s, z_{s}+\widehat{\phi}_{s}\right) \mathrm{d} w(s)+\sum_{0<t_{k}<t} C\left(t-t_{k}\right) I_{k}\left(z_{t_{k}}+\widehat{\phi}_{t_{k}}\right) \\
+\sum_{0<t_{k}<t} S\left(t-t_{k}\right) J_{k}\left(z_{t_{k}}+\widehat{\phi}_{t_{k}}\right), \quad \text { for a.e. } t \in J .
\end{array}\right.
$$

Obviously, the operator $\Psi$ has a fixed point which is equivalent to prove that $\widehat{\Psi}$ has a fixed point. Since all the functions involved in the operator are continuous therefore $\widehat{\Psi}$ is continuous. From our assumptions we have

$$
\begin{aligned}
E\left\|u_{z+\widehat{\phi}}^{a}\right\|^{2} \leq & 12 N_{W}\left\{\left\|x_{1}\right\|^{2}+N\|\phi(0)\|^{2}+\tilde{N}\|\eta\|^{2}+\tilde{N}\left(N_{1}\|\widehat{\phi}\|_{\mathcal{B}}^{2}+N_{2}\right)+a^{2} N[4 \times\right. \\
\times & \left.N_{1}\left(K_{a}^{2} q+N^{*}\right)+N_{2}\right]+\left(10 \tilde{N}+2 a^{2} N\right) N_{D}\left(q+\|\widehat{\phi}\|_{a}\right)+2 a^{2} \tilde{N}\left[4 N _ { f } \left(K_{a}^{2} q\right.\right. \\
+ & \left.\left.N^{*}\right)+\tilde{N}_{f}\right]+2 a \tilde{N} \operatorname{Tr}(Q)\left[4 N_{\sigma}\left(K_{a}^{2} q+N^{*}\right)+\tilde{N}_{\sigma}\right]+2 m N\left[4 \sum _ { k = 1 } ^ { m } \beta _ { k } \left(K_{a}^{2} q\right.\right. \\
+ & \left.\left.\left.N^{*}\right)+\sum_{k=1}^{m} \tilde{\beta}_{k}\right]+2 m \tilde{N}\left[4 \sum_{k=1}^{m} \alpha_{k}\left(K_{a}^{2} q+N^{*}\right)+\sum_{k=1}^{m} \tilde{\alpha}_{k}\right]\right\} \text { and } \\
E\left\|u_{z+\widehat{\phi}}^{a}-u_{w+\widehat{\phi}}^{a}\right\|^{2} \leq & 8 N_{W}\left\{a^{2} N N_{g}+\left(5 \tilde{N}+a^{2} N\right) N_{D}+a^{2} \tilde{N} N_{f}+a \tilde{N} T r(Q) N_{\sigma}\right. \\
& \left.+m N \sum_{k=1}^{m} \beta_{k}+m \tilde{N} \sum_{k=1}^{m} \alpha_{k}\right\}\left\|z_{t}-w_{t}\right\|_{\mathcal{B}}^{2} .
\end{aligned}
$$

Step 1: $\widehat{\Psi}\left(B_{q}\right) \subseteq B_{q}$ for some $q>0$.

We claim that there exists a positive integer $q$ such that $\widehat{\Psi}\left(B_{q}\right) \subseteq B_{q}$. If it is not true, then for each positive number $q$, there exists a function $z^{q}(\cdot) \in B_{q}$, but $\widehat{\Psi}\left(z^{q}\right) \notin B_{q}$, i.e. $\left\|\left(\widehat{\Psi} z^{q}\right)(t)\right\|^{2}>q$ for some $t \in J$. However, on the other hand from $(H 1)-(H 7)$ we have

$$
\begin{aligned}
q< & E\left\|\left(\widehat{\Psi} z^{q}\right)(t)\right\|^{2} \\
\leq & 10\left\{( 1 + 1 2 a ^ { 2 } \tilde { N } N _ { B } N _ { W } ) \left(\tilde{N}\left(N_{1}\|\widehat{\phi}\|_{\mathcal{B}}^{2}+N_{2}\right)+a^{2} N\left[4 N_{1}\left(K_{a}^{2} q+N^{*}\right)+N_{2}\right]+(10 \tilde{N}\right.\right. \\
& \left.+2 a^{2} N\right) N_{D}\left(q+\|\widehat{\phi}\|_{a}\right)+2 a^{2} \tilde{N}\left[4 N_{f}\left(K_{a}^{2} q+N^{*}\right)+\tilde{N}_{f}\right]+2 a \tilde{N} \operatorname{Tr}(Q)\left[4 N_{\sigma}\left(K_{a}^{2} q+N^{*}\right)\right. \\
& \left.\left.+\tilde{N}_{\sigma}\right]+2 m N\left[4 \sum_{k=1}^{m} \beta_{k}\left(K_{a}^{2} q+N^{*}\right)+\sum_{k=1}^{m} \tilde{\beta}_{k}\right]+2 m \tilde{N}\left[4 \sum_{k=1}^{m} \alpha_{k}\left(K_{a}^{2} q+N^{*}\right)+\sum_{k=1}^{m} \tilde{\alpha}_{k}\right]\right) \\
& \left.+12 a^{2} \tilde{N} N_{B} N_{W}\left(\left\|x_{1}\right\|^{2}+N\|\phi(0)\|^{2}+\tilde{N}\|\eta\|^{2}\right)\right\} \\
\leq & L+10\left\{4 K _ { a } ^ { 2 } q ( 1 + 1 2 a ^ { 2 } \tilde { N } N _ { B } N _ { W } ) \left(a^{2} N N_{1}+\frac{N_{D}}{2 K_{a}^{2}}\left(5 \tilde{N}+a^{2} N\right)+2 a^{2} \tilde{N} N_{f}\right.\right. \\
& \left.\left.+2 a \tilde{N} \operatorname{Tr}(Q) N_{\sigma}+2 m\left[N \sum_{k=1}^{m} \beta_{k}+\tilde{N} \sum_{k=1}^{m} \alpha_{k}\right]\right)\right\}
\end{aligned}
$$


where $L$ is independent of $q$. Dividing both sides by $q$ and as $q \rightarrow \infty$ we have

$$
\begin{aligned}
10\{(1+ & \left.12 a^{2} \tilde{N} N_{B} N_{W}\right) 4 K_{a}^{2}\left(a^{2} N N_{1}+\frac{N_{D}}{2 K_{a}^{2}}\left(5 \tilde{N}+a^{2} N\right)+2 a^{2} \tilde{N} N_{f}+2 a \tilde{N} T r(Q) N_{\sigma}\right. \\
+ & \left.\left.2 m\left[N \sum_{k=1}^{m} \beta_{k}+\tilde{N} \sum_{k=1}^{m} \alpha_{k}\right]\right)\right\} \geq 1 .
\end{aligned}
$$

This contradicts (3.1). Hence $\widehat{\Psi}\left(B_{q}\right) \subseteq B_{q}$, for some positive number $q$.

Step 2: $\widehat{\Psi}: \mathcal{B}_{a}^{0} \rightarrow \mathcal{B}_{a}^{0}$ is a contraction mapping.

Let $z, w \in \mathcal{B}_{a}^{0}$ then we have

$$
\begin{aligned}
& E\|\widehat{\Psi} z(t)-\widehat{\Psi} w(t)\|^{2} \leq E\left\|\int_{0}^{t} C(t-s)\left(g\left(s, z_{s}+\widehat{\phi}_{s}\right)-g\left(s, w_{s}+\widehat{\phi}_{s}\right)\right) \mathrm{d} s\right\|^{2} \\
& +E \| \sum_{i=0}^{j-1}\left[S\left(t-t_{i+1}\right) \mathcal{D}\left(z\left(t_{i+1}^{-}\right)+\widehat{\phi}\left(t_{i+1}^{-}\right)-w\left(t_{i+1}^{-}\right)-\widehat{\phi}\left(t_{i+1}^{-}\right)\right)\right. \\
& \left.-S\left(t-t_{i}\right) \mathcal{D}\left(z\left(t_{i}^{+}\right)+\widehat{\phi}\left(t_{i}^{+}\right)-w\left(t_{i}^{+}\right)-\widehat{\phi}\left(t_{i}^{+}\right)\right)\right] \|^{2} \\
& +E\left\|S\left(t-t_{j}\right) \mathcal{D}\left(z\left(t_{j}^{+}\right)+\widehat{\phi}\left(t_{j}^{+}\right)-w\left(t_{j}^{+}\right)-\widehat{\phi}\left(t_{j}^{+}\right)\right)\right\|^{2} \\
& +E\left\|\int_{0}^{t} S(t-s) B\left[u_{z+\widehat{\phi}}^{a}-u_{w+\widehat{\phi}}^{a}(s)\right] \mathrm{d} s\right\|^{2} \\
& +E\left\|\int_{0}^{t} C(t-s) \mathcal{D}(z(s)+\widehat{\phi}(s)-w(s)-\widehat{\phi}(s)) \mathrm{d} s\right\|^{2} \\
& +E \| \int_{0}^{t} S\left(t-s\left[f\left(s, z_{s}+\widehat{\phi}_{s}\right)-f\left(s, w_{s}+\widehat{\phi}_{s}\right)\right] \mathrm{d} s \|^{2}\right. \\
& +E\left\|\int_{0}^{t} S(t-s)\left[\sigma\left(s, z_{s}+\widehat{\phi}_{s}\right)-\sigma\left(s, w_{s}+\widehat{\phi}_{s}\right)\right] \mathrm{d} w(s)\right\|^{2} \\
& +E\left\|\sum_{0<t_{k}<t} C\left(t-t_{k}\right)\left[I_{k}\left(z_{t_{k}}+\widehat{\phi}_{t_{k}}\right)-I_{k}\left(w_{t_{k}}+\widehat{\phi}_{t_{k}}\right)\right]\right\|^{2} \\
& +E\left\|\sum_{0<t_{k}<t} S\left(t-t_{k}\right)\left[J_{k}\left(z_{t_{k}}+\widehat{\phi}_{t_{k}}\right)-J_{k}\left(w_{t_{k}}+\widehat{\phi}_{t_{k}}\right)\right]\right\|^{2} \\
& \leq 9\left\{( 1 + 8 a ^ { 2 } \tilde { N } N _ { B } N _ { W } ) \left(a^{2} N N_{g}+\left(5 \tilde{N}+a^{2} N\right) N_{D}+a^{2} \tilde{N} N_{f}\right.\right. \\
& \left.\left.+a \tilde{N} \operatorname{Tr}(Q) N_{\sigma}+m N \sum_{k=1}^{m} \beta_{k}+m \tilde{N} \sum_{k=1}^{m} \alpha_{k}\right)\right\}\left\|z_{t}-w_{t}\right\|_{\mathcal{B}}^{2} \\
& \leq 9\left\{( 1 + 8 a ^ { 2 } \tilde { N } N _ { B } N _ { W } ) \left(a^{2} N N_{g}+\left(5 \tilde{N}+a^{2} N\right) N_{D}+a^{2} \tilde{N} N_{f}\right.\right. \\
& \left.\left.+a \tilde{N} \operatorname{Tr}(Q) N_{\sigma}+m N \sum_{k=1}^{m} \beta_{k}+m \tilde{N} \sum_{k=1}^{m} \alpha_{k}\right)\right\} \\
& \times\left[K_{a}^{2} \sup _{0 \leq s \leq a} E\|z(s)-w(s)\|^{2}+M_{a}^{2}\left\|z_{0}-w_{0}\right\|_{\mathcal{B}}^{2}\right] \\
& \leq 9 K_{a}^{2}\left\{( 1 + 8 a ^ { 2 } \tilde { N } N _ { B } N _ { W } ) \left(a^{2} N N_{g}+\frac{1}{K_{a}^{2}}\left(5 \tilde{N}+a^{2} N\right) N_{D}+a^{2} \tilde{N} N_{f}\right.\right. \\
& \left.\left.+a \tilde{N} \operatorname{Tr}(Q) N_{\sigma}+m N \sum_{k=1}^{m} \beta_{k}+m \tilde{N} \sum_{k=1}^{m} \alpha_{k}\right)\right\} \sup _{0 \leq s \leq a} E\|z(s)-w(s)\|^{2} \\
& \leq \nu \sup _{0 \leq s \leq a} E\|z(s)-w(s)\|^{2},
\end{aligned}
$$


since $\nu<1$ by $(H 7)$ and we have used the fact that $z_{0}=0, w_{0}=0$. Taking the supremum over $t$, we get

$$
\|\widehat{\Psi} z-\widehat{\Psi} w\|_{a}^{2} \leq \nu\|z-w\|_{a}^{2},
$$

and so $\widehat{\Psi}$ is a contraction. Hence by Banach fixed point theorem there exists a unique fixed point $x \in \mathcal{B}_{a}$ such that $(\Psi x)(t)=x(t)$. This fixed point is then the solution of the system (2.1) and clearly, $x(a)=(\Psi x)(a)=x_{1}$ which implies that the system $(2.1)$ is controllable on $J$.

\section{Example}

In this section, we consider an application for the abstract result developed in Section 3 . In the sequel, $H=K=U=L^{2}([0, \pi]) ; \mathcal{B}=\mathcal{P C}_{0} \times \mathcal{L}^{2}(\rho, H)$ and $A: D(A) \subseteq H \rightarrow H$ is the map defined by $A f=f^{\prime \prime}$ with domain $D(A)=\left\{f \in H: f^{\prime \prime} \in H, f(0)=f(\pi)=0\right\}$. It is well-known that $A$ is the infinitesimal generator of a strongly continuous cosine function $(C(t))_{t \in \mathbb{R}}$ on $H$. Furthermore, $A$ has a discrete spectrum, the eigenvalues are $-n^{2}, n \in \mathbb{N}$, with corresponding eigenvectors $z_{n}(\theta)=\left(\frac{2}{\pi}\right)^{1 / 2} \sin (n \theta)$; the set $\left\{z_{n}: n \in \mathbb{N}\right\}$ is an orthonormal basis of $H$ and the following properties hold:

(a) For $\varphi \in D(A), A \varphi=-\sum_{n=1}^{\infty} n^{2}<\varphi, z_{n}>z_{n}$.

(b) For $\varphi \in H, C(t) \varphi=\sum_{n=1}^{\infty} \cos (n t)<\varphi, z_{n}>z_{n}$ and $S(t) \varphi=\sum_{n=1}^{\infty} \frac{\sin (n t)}{n}<\varphi, z_{n}>z_{n}$. Consequently $\|C(t)\|=\|S(t)\| \leq 1$, for all $t \in \mathbb{R}$ and $S(t)$ is compact for every $t \in \mathbb{R}$.

(c) If $\Phi$ is the group of translations on $H$ defined by $\Phi(t) x(\xi)=\tilde{x}(\xi+t)$, where $\tilde{x}$ is the extension of $x$ with period $2 \pi$, then $C(t)=\frac{1}{2}(\Phi(t)+\Phi(-t))$ and $A=B^{2}$, where $B$ is the generator of $\Phi$ and $E=\left\{x \in H^{1}(0, \pi): x(0)=x(\pi)=0\right\}$ (see [27] for details). In particular, we observe that the inclusion $\iota: E \rightarrow H$ is compact.

Consider the following impulsive neutral second order partial differential equation

$$
\begin{aligned}
\partial\left[\frac{\partial}{\partial t} u(t, \tau)-\right. & \left.\int_{-\infty}^{t} \int_{0}^{\pi} b(t-s, \vartheta, \tau) u(s, \vartheta) \mathrm{d} \vartheta \mathrm{d} s\right]=\left[\frac{\partial^{2}}{\partial \tau^{2}} u(t, \tau)+\alpha \frac{\partial}{\partial t} u(t, \tau)\right. \\
& \left.+\int_{0}^{\pi} \beta(s) \frac{\partial}{\partial t} u(t, s) \mathrm{d} s+\mu(t, \tau)+\int_{-\infty}^{t} c(t-s) u(s, \tau) \mathrm{d} s\right] \partial t \\
& +\int_{-\infty}^{t} G(t-s) u(s, \tau) \mathrm{d} w(s), \quad t \in J=[0, a], \tau \in[0, \pi], t \neq t_{k} \\
u(t, 0)= & u(t, \pi)=0, \quad t \in J, \\
u(s, \tau)= & \varphi(s, \tau), \frac{\partial}{\partial t} u(0, \tau)=\xi(\tau), s \in(-\infty, 0], \tau \in[0, \pi] \\
\triangle u\left(t_{k}, \tau\right)= & \int_{-\infty}^{t_{k}} \gamma_{k}\left(t_{k}-s\right) u(s, \tau) \mathrm{d} s, k=1,2, \cdots, m \\
\triangle u^{\prime}\left(t_{k}, \tau\right)= & \int_{-\infty}^{t_{k}} \tilde{\gamma}_{k}\left(t_{k}-s\right) u(s, \tau) \mathrm{d} s, k=1,2, \cdots, m
\end{aligned}
$$

where we assume that $\varphi \in \mathcal{B}$, with the identification $\varphi(s)(\tau)=\varphi(s, \tau), \varphi(0, \cdot) \in H^{1}([0, \pi]), \xi \in H$ and $0<t_{1}<\ldots<t_{m}<a$. Here $\alpha$ is a prefixed real number and $\beta \in L^{2}([0, \pi])$. To treat this system, we assume that the functions $b, c, G, \gamma_{k}, \tilde{\gamma}_{k}$ satisfy the following conditions:

(i) The functions $b(s, \nu, \tau), \frac{\partial}{\partial \tau} b(s, \nu, \tau)$ are continuous and measurable, $b(s, \nu, \pi)=b(s, \nu, 0)$ $=0$ for every $(s, \nu) \in(-\infty, 0] \times J$ and

$$
N_{g}=\max \left\{\left[\int_{0}^{\pi} \int_{-\infty}^{0} \int_{0}^{\pi} \frac{1}{\rho(s)}\left(\frac{\partial^{i} b(s, \vartheta, \tau)}{\partial \tau^{i}}\right)^{2} \mathrm{~d} \vartheta \mathrm{d} s \mathrm{~d} \tau\right]^{\frac{1}{2}}: i=0,1\right\}<\infty
$$


(ii) The functions $c(\cdot), G(\cdot), \gamma_{k}, \tilde{\gamma}_{k} k=1, \cdots, m$ are continuous, $N_{f}=\left(\int_{-\infty}^{0} \frac{c^{2}(-s)}{\rho(s)} \mathrm{d} s\right)^{\frac{1}{2}}, N_{\sigma}=\left(\int_{-\infty}^{0} \frac{G^{2}(-s)}{\rho(s)} \mathrm{d} s\right)^{\frac{1}{2}}$, $N_{I_{k}}=\left(\int_{-\infty}^{0} \frac{\gamma_{k}^{2}(-s)}{\rho(s)} \mathrm{d} s\right)^{\frac{1}{2}}$ and $N_{J_{k}}=\left(\int_{-\infty}^{0} \frac{\tilde{\gamma}_{k}^{2}(-s)}{\rho(s)} \mathrm{d} s\right)^{\frac{1}{2}}$, for every $k=1,2, \cdots, m$ are finite.

We now define the functions $\mathcal{D}: H \rightarrow H, f, g: J \times \mathcal{B} \rightarrow H \sigma: J \times \mathcal{B} \rightarrow \mathcal{L}_{Q}(K, H)$, and $I_{k}, J_{k}: \mathcal{B} \rightarrow H$ by

$$
\begin{gathered}
\mathcal{D} \psi(\tau)=\alpha \psi(t, \tau)+\int_{0}^{\pi} \beta(s) \psi(t, s) \mathrm{d} s, g(\psi)(\tau)=\int_{-\infty}^{0} \int_{0}^{\pi} b(-s, \vartheta, \tau) \psi(s, \vartheta) \mathrm{d} \vartheta \mathrm{d} s, \\
f(\psi)(\tau)=\int_{-\infty}^{0} c(-s) \psi(s, \tau) \mathrm{d} s, \sigma(\psi)(\tau)=\int_{-\infty}^{0} G(-s) \psi(s, \tau) \mathrm{d} s \\
I_{k}(\psi)(\tau)=\int_{-\infty}^{0} \gamma_{k}(-s) \psi(s, \tau) \mathrm{d} s, J_{k}(\psi)(\tau)=\int_{-\infty}^{0} \tilde{\gamma}_{k}(-s) \psi(s, \tau) \mathrm{d} s .
\end{gathered}
$$

Assume the bounded linear operator $B: L^{2}([0, \pi]) \rightarrow H$ and is defined by

$$
(B u)(t)(\tau)=\mu(t, \tau), \quad \tau \in[0, \pi] .
$$

Also, the linear operator $W: L^{2}(J, U) \rightarrow H$ is given by

$$
(W u)(\tau)=\sum_{n=1}^{\infty} \int_{0}^{\pi} \frac{1}{n} \sin n s\left(\mu(s, \tau), e_{n}\right) e_{n} \mathrm{~d} s, \tau \in[0, \pi]
$$

has an inverse operator $W^{-1}$ defined on $L^{2}(J, U) \backslash K e r W$ and satisfies $(H 1)$. Under these conditions, the maps $\mathcal{D}, f, g, \sigma, I_{k}, J_{k}$ are bounded linear operators, $\|\mathcal{D}\|_{\mathcal{L}(X)} \leq\|\alpha\|+\|\beta\|_{L^{2}(0, a)},\|f\| \leq N_{f},\|g\| \leq N_{g}$, $\|\sigma\| \leq N_{\sigma},\left\|I_{k}\right\| \leq N_{I_{k}}$ and $\left\|J_{k}\right\| \leq N_{J_{k}}$. With this choice of $A, \mathcal{D}, B, f, g, \sigma, I_{k}, J_{k}$, the equation (4.1) can be written in the abstract formulation of the system (2.1). Further all the assumptions $(H 2)-(H 7)$ are satisfied and hence by Theorem 3.1, the system (4.1) is controllable on $J$.

Acknowledgments. The second author is thankful to UGC, New Delhi for providing BSR- Fellowship during 2010.

\section{References}

1. E. Emmrich and M. Thalhammer, Convergence of a time discretisation for doubly nonlinear evolution equations of second order, Foundations of Computational Mathematics, 2010, 10: 171-190.

2. E. Emmrich and D. Siska, Full discretisation of second-order nonlinear evolution equations: strong convergence and applications, Computational Methods in Applied Mathematics, 2011, 11: 441-459.

3. L.A. Medeiros and M. Milla Miranda, On a nonlinear wave equation with damping, Revista Mathematica de la, 1990, 3: 213-231.

4. C.C. Travis and G.F. Webb, Compactness, regularity and uniform continuity properties of strongly continuous cosine families, Houston Journal of Mathematics, 1977, 3: 555-567.

5. C.C. Travis and G.F. Webb, Cosine families and abstract nonlinear second order differential equations, Acta Mathematica Academiae Scientiarum Hungaricae, 1978, 32: 75-96.

6. Y. Hino, S. Murakami and T. Naito, Functional-Differential Equations with Infinite Delay, Lecture Notes in Mathematics, 1473, Springer, Berlin, 1991.

7. P. Balasubramaniam and P. Muthukumar, Approximate controllability of second-order stochastic distributed implicit functional differential systems with infinite delay, Journal of Optimization Theory and Applications, 2009, 143: 225-244.

8. J.K. Hale and J. Kato, Phase space for retarded equations with infinite delay, Funkcialaj Ekvacioj-Serio Internacia, 1978, 21: 11-41.

9. E. Hernandez and H.R. Henriquez, Existence results for second order partial neutral functional differential equations, Dynamics of Continuous, Discrete and Impulsive Systems. Series A, 2008, 15(5): 645-670.

10. L. Hu and Y. Ren, Existence results for impulsive neutral stochastic functional integrodifferential equations with infinite delays, Acta Applicandae Mathematicae, 2010, 111: 303-317. 
11. Y. Ren and D.D. Sun, Second-order neutral stochastic evolution equations with infinite delay under Caratheodory conditions, Journal of Optimization Theory and Applications, 2010, 147: 569-582.

12. R. Ye and G. Zhang, Neutral functional differential equations of second-order with infinite delays, Electronic Journal of Differential Equations, 2010, 36: 1-12.

13. V. Lakshmikantham, D.D. Bainov and P.S. Simeonov, Theory of Impulsive Differential Equations, World Scientific, Singapore, 1989.

14. A.M. Samoilenko and N.A. Perestyuk, Impulsive Differential Equations, World Scientific, Singapore, 1995.

15. K. Balachandran and S. Anthoni, Controllability of second-order semilinear neutral functional differential systems in Banach spaces, Computers and Mathematics with Applications, 2001, 41: 1223-1235.

16. K. Balachandran, D.G. Park, and S. Anthoni, Existence of solutions of abstract nonlinear second-order neutral functional integrodifferential equations, Computers and Mathematics with Applications, 2003, 46: 1313-1324.

17. E. Hernandez, H.R. Henriquez and M.A. Mckibben, Existence results for abstract impulsive second-order neutral functional differential equations, Nonlinear Analysis, 2009, 70: 2736-2751.

18. N.I. Mahmudov and M.A. McKibben, Approximate controllability of second-order neutral stochastic evolution equations, Dynamics of Continuous, Discrete and Impulsive Systems. Series B, 2006, 13(5): 619-634.

19. M.A. McKibben, Second-order neutral stochastic evolution equations with heredity, Journal of Applied Mathematics and Stochastic Analysis, 2004, 2: 177-192.

20. R. Sakthivel, N.I. Mahmudov and J.H. Kim, On controllability of second order nonlinear impulsive differential systems, Nonlinear Analysis, 2009, 71: 45-52.

21. E. Hernandez, K. Balachandran and N. Annapoorani, Existence results for a damped second order abstract functional differential equation with impulses, Mathematical and Computer Modelling, 2009, 50: 1583-1594.

22. N.I. Mahmudov and M.A. McKibben, Abstract second order damped Mckean-Vlasov stochastic evolution equations, Stochastic Analysis and Applications, 2006, 24: 303-328.

23. M.A. McKibben, Second-order damped functional stochastic evolution equations in Hilbert spaces, Dynamic Systems and Applications, 2003, 12: 467-488.

24. G. Arthi and K. Balachandran, Controllability of damped second-order impulsive neutral functional differential systems with infinite delay, Journal of Optimization Theory and Applications, 2012, 152: 799-813.

25. G. Da Prato and J. Zabczyk, Stochastic Equations in Infinite Dimensions, Cambridge University Press, Cambridge, 1992.

26. J. Kisynski, On cosine operator functions and one parameter group of operators, Studia Mathematica, 1972, 49: 93-105.

27. H.O. Fattorini, Second Order Linear Differential Equations in Banach Spaces, North-Holland, Amsterdam, 1985.

28. J.P. Dauer and N.I. Mahmudov, Controllability of stochastic semilinear functional differential equations in Hilbert spaces, Journal of Mathematical Analysis and Applications, 2004, 290: 373-394. 